\title{
Diagnostic defaulters: an overlooked aspect in the Indian Revised National Tuberculosis Control Program
}

\author{
Jagdish Rawat ${ }^{1}$, Debasis Biswas ${ }^{2}$, Girish Sindhwani ${ }^{1}$, Vikas Kesharwani ${ }^{1}$, Victor Masih ${ }^{2}$, \\ Bhupendra Singh Chauhan².
}

${ }^{1}$ Department of Pulmonary Medicine Himalayan Institute of Medical Sciences, Swami Ram Nagar, Jolly Grant, Dehradun, Uttarakhand, India

${ }^{2}$ Department of Microbiology, Himalayan Institute of Medical Sciences, Swami Ram Nagar, Jolly Grant, Dehradun, Uttarakhand, India

\begin{abstract}
Introduction: The Revised National Tuberculosis Control Program (RNTCP) aims to achieve detection of 70\% of new smear-positive patients in a community and to cure $85 \%$ of such patients. Though an elaborate recording and reporting system is in place to monitor the success of the program, no record of patients who do not complete the diagnostic process is currently maintained. The present study, performed in a tertiary care hospital, attempted to estimate the proportion of this group of patients, who were labeled "diagnostic defaulters."

Methodology: This prospective observational study was conducted over one year on consecutive patients presenting with cough of more than two weeks' duration. A total of two sputum samples were obtained from each patient, including a spot sample and a morning sample collected on the following day. Zeihl-Neelsen staining, reporting and external quality assessment of smear-microscopy was done per RNTCP guidelines.

Results: Of the 2,349 patients recruited, 175 defaulted on the second day. The positivity rates for the spot and morning samples were $18.3 \%$ and $18.9 \%$, respectively ( $\mathrm{p}>0.001$ ). Of the 175 defaulters, 31 were found to be smear-positive, thereby implying that the proportion of diagnostic defaulters was $17.7 \%$. All 21 diagnostic defaulters contacted by telephone were found to be unaware of their smear-positive status. Conclusion: The high proportion of diagnostic defaulters, the majority of whom were unaware of their sputum-positive status, emphasizes the need to recognize the importance of this group of patients to better control tuberculosis.
\end{abstract}

Key words: defaulter; smear-microscopy; pulmonary tuberculosis

J Infect Dev Ctries 2012; 6(1):20-22.

(Received 28 January 2011 - Accepted 06 March 2011)

Copyright (C) 2012 Rawat et al. This is an open-access article distributed under the Creative Commons Attribution License, which permits unrestricted use, distribution, and reproduction in any medium, provided the original work is properly cited.

\section{Introduction}

The success of the Indian Revised National Tuberculosis Control Program (RNTCP) program is evaluated by a number of outcome measures. These include the annualized case detection rate for new smear-positive cases, the proportion of new sputumpositive patients out of the total number of new cases of pulmonary tuberculosis (TB), the smear conversion rate, cured rate, treatment completed rate, treatment defaulter rate, and the rate of patient deaths during treatment. The program has an inbuilt mechanism of regular recording and reporting of these performance indicators at every level of the healthcare system. However, this system does not include a report on the number of smear-positive patients who default on the second day of microscopic examination. Since the routine practice for microscopy centers in India is to dispatch smear- examination reports only after all the smears of a particular patient have been examined, these patients remain unaware of their smear status and are not referred for treatment. The data on this group of smear-positive patients is limited, though there have been a few studies on "initial defaulters" [1-5], i.e., TB patients who have been diagnosed but have not initiated treatment [6]. In view of the potential impact of this group on the transmission of $\mathrm{TB}$ and the success of the TB control programs, this study aimed to determine the magnitude of their proportion among the patients attending a typical Designated Microscopy Center (DMC) attached to a tertiary care teaching hospital. The term "diagnostic defaulters" refers to these patients to differentiate them from initial defaulters. 
Figure 1. Relative yield of spot and morning samples

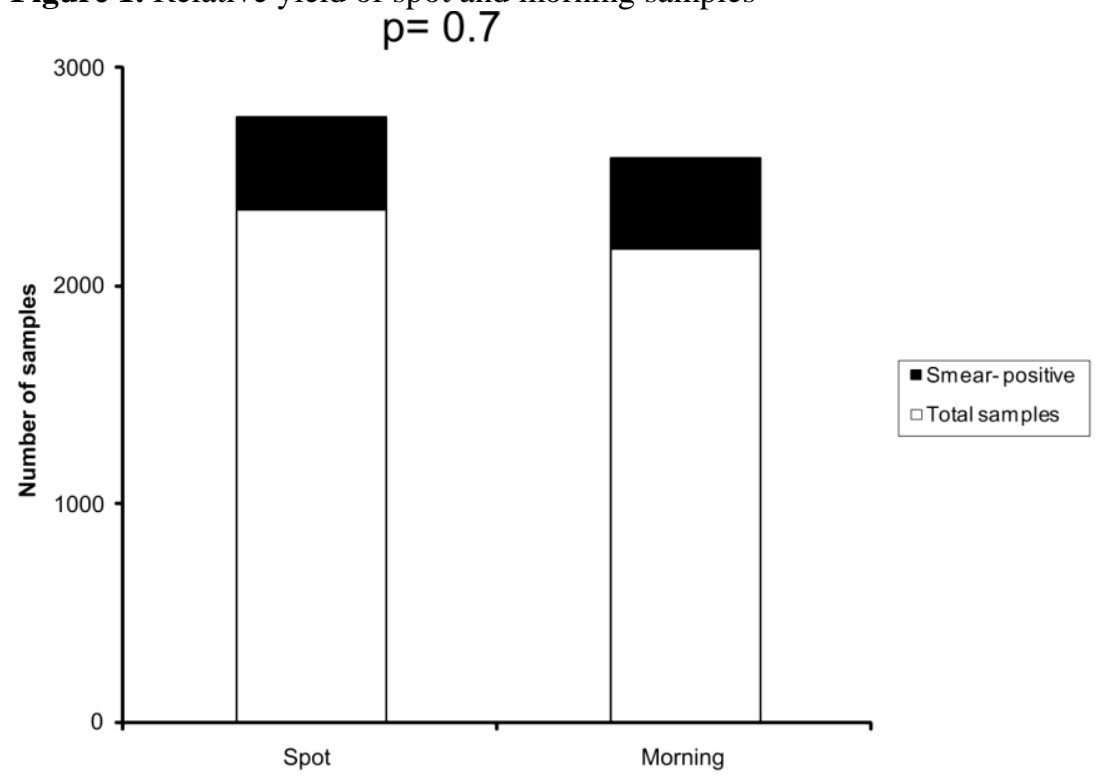

\section{Methodology}

This prospective cross-sectional study was conducted over a period of one year from June 2009 to May 2010 in the DMC attached to the Pulmonary Medicine OPD of the Himalayan Institute of Medical Sciences, which operates within the operational protocol of RNTCP. The study protocol was approved by the institutional ethics committee. All self-reporting chest symptomatic patients who had a cough for over two weeks were recruited for the study. A total of two sputum samples were requested from each patient, including a spot sample (a) and a morning sample collected on the following day (b). The sputum samples were subjected to smear microscopy by Zeihl Neelsen staining and reported per RNTCP guidelines [7]. External quality assessment of smear microscopy was performed monthly using standard methods recommended by the RNTCP regarding on-site evaluation of the microscopy center by a trained lab supervisor and random blind re-checking of slides. A patient was labeled a diagnostic defaulter if he failed to report on the second day and was found to be smear positive on examination of the spot sample. Attempts were made to contact the defaulters by telephone to offer counseling, and to determine if they were aware of their smear-positive status and to learn the reason for their defaulting.

The diagnostic yields of the spot and morning samples were compared using the $\mathrm{z}$ test for two proportions; a p-value less than 0.05 was considered to be significant.

\section{Results}

A total of 2,349 pulmonary TB suspects were recruited during the study period. Of them, 175 patients failed to report on the second day. Of the total number of recruited patients, $466(19.8 \%)$ were found to be sputum positive. Among the patients who submitted a spot sample, $429(18.3 \%)$ were smear positive; of the 2,174 morning samples, 411 (18.9\%) were smear positive $(\mathrm{p}=0.7)$ (Figure). The morning sample did not offer any significant benefit over the spot sample. Among the 175 patients who dropped out of the study, $31(17.7 \%)$ were found to be smear positive based on the examination of the spot sample, thereby implying that the proportion of diagnostic defaulters in this study was $17.7 \%$. Telephone contact was established with 21 of these 31 diagnostic defaulters $(67.7 \%)$, all of whom were found to be unaware of their smear-positive status. When questioned about the reason for their defaulting, seven patients cited long distance as the reason. The other patients demonstrated a lack of motivation for making a second visit and indicated that vocational priorities prevented them from visiting the microscopy center on the second day.

\section{Discussion}

This study found that of the drop-out patients in the DMC attached to a tertiary care teaching hospital, $17.7 \%$ were smear positive and were termed diagnostic defaulters. The reporting and monitoring system in RNTCP does not include a record of this group of patients; this is the first prospective study 
that has attempted to determine a quantitative estimate of this group. Most of the diagnostic defaulters were unaware of their smear-positive status and thus pose a remarkable threat to their contacts and to the success of the control program.

The study found that spot and morning samples do not differ significantly in their diagnostic yields. Moreover, it has been reported by the present authors and some other researchers that a one-day protocol for smear microscopy, including two spot samples collected at one hour intervals, achieves similar diagnostic sensitivity compared to the recommended two-day protocol, which includes a spot and a morning sample [8-10]. In view of these findings, the authors suggest that to reduce the number of diagnostic defaulters, the current system of sputum examination and reporting should be partially modified to accommodate an alternative plan wherein patients found to be smear positive in the first spot sample should be directly referred for treatment on that day. In the present system, they are required to report to the microscopy center the following day with their morning sputum sample; their sputumpositivity status is disclosed only after an examination of the second sample.

The high proportion of diagnostic defaulters in this study could be due to the fact that it was conducted in a tertiary care center, which attends to patients from distant regions. These findings are likely to be duplicated in other medical colleges. This group of patients, who are likely to be encountered in significant numbers in tertiary centers and district hospitals, needs to be recognized and included in the regular reporting and monitoring schedule of RNTCP. In view of the similarity of findings observed between the one day protocol and two day protocol of sputum collection, patients coming from distant places in tertiary centers might be offered the choice of submitting two spot samples over a period of one hour and thereby completing the diagnostic exercise and collecting their microscopy reports in the same day. This protocol may also be offered to patients in regions with hilly and difficult terrains and also to patients who live in financially weaker sections who have to forego their wages to attend the centre for an additional day. This approach would reduce the load of diagnostic defaulters without significantly compromising case-detection rates.

The proportion of diagnostic defaulters is significantly high in Indian tertiary centers and can be minimized by either referring patients for treatment on the basis of one positive spot sample or by offering the choice of the one day protocol to a select group of patients who are likely to default.

\section{References}

1. Reed JB, McCausland R, Elwood JM (1990) Default in the outpatient treatment of tuberculosis in two hospitals in Northern India. J Epidemiol Community Health 44: 20-23.

2. Vijay S, Balasangameswara VH, Jagannatha PS, Saroja VN, Kumar P (2003) Defaults among tuberculosis patients treated under DOTS in Bangalore city: A search for solution. Indian J Tuberc 50: 185-195.

3. Botha E, den Boon S, Lawrence KA, Reuter H, Verver S, Lombard CJ, Dye C, Enarson DA, Beyers N (2008) From suspect to patient: tuberculosis diagnosis and treatment initiation in health facilities in South Africa. Int $\mathbf{J}$ Tuberc Lung Dis 12: 936-941.

4. Botha E, Den Boon S, Verver S, Dunbar R, Lawrence KA, Bosman M, Enarson DA, Toms I, Beyers N (2008) Initial default from tuberculosis treatment: how often does it happen and what are the reasons? Int J Tuberc Lung Dis 12: 820-823.

5. Sai Babu B, Satyanarayana AV, Venkateshwaralu G, Ramakrishna U, Vikram P, Sahu S, Wares F, Dewan PK, Santosha K, Jyoti J, Srinath S, Chethana R, Neelima T, Vinod P, Yogesh M, Chauhan LS (2008) Initial default among diagnosed sputum smear-positive pulmonary tuberculosis patients in Andhra Pradesh, India. Int J Tuberc Lung Dis 12: 1055-1058.

6. Harries AD, Rusen ID, Chiang CY, Hinderaker SG, Enarson DA (2009) Registering initial defaulters and reporting on their treatment outcomes. Int J Tuberc Lung Dis 13: 801803.

7. Directorate General of Health Services, Ministry of Health and Family Welfare. Tuberculosis control-India. [Accessed $1^{\text {st }}$ May 2009] Available from URL: http://www.tbcindia.org.

8. Hirao S, Yassin MA, Khamofu HG, Lawson L, Cambanis A, Ramsay A, Cuevas LE (2007) Same day smears in the diagnosis of tuberculosis. Trop Med Int Health 12: 14591463.

9. Cambanis A, Yassin MA, Ramsay A, Squire SB, Arbide I, Cuevas LE (2006) A one day method for the diagnosis of pulmonary tuberculosis in rural Ethiopia. Int J Tuberc Lung Dis 10: 230-232.

10. Rawat J, Biswas D, Sindhwani G, Masih V (2010) An alternative one-day smear microscopy protocol for the diagnosis of pulmonary tuberculosis. Respirology 15: 11271130 .

\section{Corresponding Author}

Dr Debasis Biswas

Professor, Dept of Microbiology

Himalayan Institute of Medical Sciences

Swami Ram Nagar, Jolly Grant

Dehradun, Uttarakhand, India 248140

Telephone: 91-135-2471575; 919411362351

Fax: 91-135-2471317

Email: dbiswas71@rediffmail.com

Conflict of interests: No conflict of interests is declared. 